\title{
Erratum: Shape of the acoustic gravitational wave power spectrum from a first order phase transition [Phys. Rev. D 96, 103520 (2017)]
}

\author{
Mark Hindmarsh, Stephan J. Huber, Kari Rummukainen, and David J. Weirø
}

(Received 14 March 2020; accepted 18 March 2020; published 9 April 2020)

DOI: $10.1103 /$ PhysRevD.101.089902

There is a factor of 3 missing from the right-hand side of Eq. (39), which should read

$$
A_{\mathrm{est}} \simeq 2.061 \Gamma^{2} \bar{U}_{\mathrm{f}}^{4} \tilde{\Omega}_{\mathrm{gw}}^{R_{*}}
$$

Without this additional factor of 3 this expression is not consistent with Eqs. (21) and (23), nor with Ref. [21]. However, the values of $A_{\text {est }}$ in Table $\mathrm{V}$ are correct and include this factor.

This factor of 3 is, in turn, missing from Eq. (45), which should read (note that the original equation also truncated the numerical coefficient as 0.68 rather than 0.687 )

$$
\frac{d \Omega_{\mathrm{gw}, 0}}{d \ln (f)}=2.061 F_{\mathrm{gw}, 0} \Gamma^{2} \bar{U}_{\mathrm{f}}^{4}\left(H_{\mathrm{n}} R_{*}\right) \tilde{\Omega}_{\mathrm{gw}} C\left(\frac{f}{f_{\mathrm{p}, 0}}\right) .
$$

We also wish to highlight that this formula is written without the customary factors of the reduced Hubble constant $h^{2}$ on each side. This itself does not affect the validity of the equation and indeed allows us to select a value of $h$ for comparison with a specific precomputed gravitational wave energy density sensitivity curve.

Note also that in the paragraph below Eq. (45) the exponent in the value for $\tilde{\Omega}_{\mathrm{gw}}$ is incorrect. The expression should $\operatorname{read} \tilde{\Omega}_{\mathrm{gw}}=1.2 \times 10^{-2}$.

The SNR contours in Fig. 9 were produced using Eq. (45). The above factor of 3 [mistakenly absent from Eq. (45)] was included but the factor of 0.68[7] [present in Eq. (45)] was excluded, and there was an additional erroneous factor of 2 . This omission means the correct predicted SNR is a factor of $0.687 \times 0.5 \approx 0.34$ lower than in the original plot.

Figure 1 below is a corrected version of Fig. 9 that takes account of the numerical errors explained above.

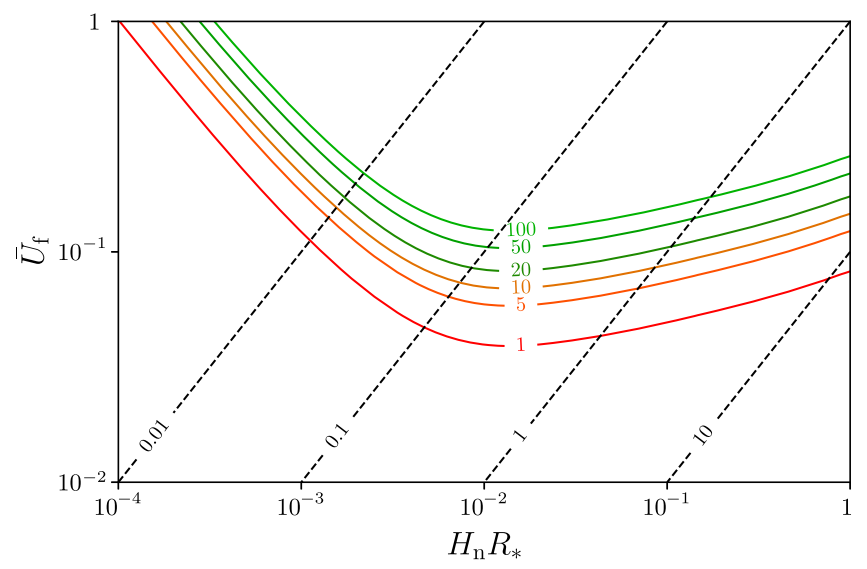

FIG. 1. Corrected version of Fig. 9, corresponding to Eq. (2) above. The missing factor of 3 in Eq. (45) was already included, but the 0.687 numerical prefactor was missing. Furthermore, an additional factor of 2 was mistakenly present. The overall effect was to reduce the SNR for a given parameter choice by a factor of approximately 0.34 compared to the original figure. Note that the mission profile for LISA used here is no longer current, so this plot is for qualitative comparison with Fig. 9 only. 
However, the mission profile of LISA has changed substantially since this paper was published, with a longer expected mission duration and a modified sensitivity curve. This will further change the predicted SNR curves. The plots in the following referenced paper [1] are free of the errors discussed in the erratum, and use the most up-to-date mission profile. ${ }^{1}$

\section{ACKNOWLEDGMENTS}

We are grateful to Aleksandr Azatov, Francesco Sgarlata and Daniele Barducci for bringing this to our attention.

[1] C. Caprini et al., Detecting gravitational waves from cosmological phase transitions with LISA: An update, J. Cosmol. Astropart. Phys. 03 (2020) 024.

\footnotetext{
${ }^{1}$ The parameter space can also be explored at ptplot.org.
} 